\title{
Methotrexate for refractory prurigo nodularis
}

\author{
Mariam Al Zaabi', Mohammad Al Suwaiji², Manal Nasir ${ }^{3}$
}

\begin{abstract}
${ }^{1}$ Department of Dermatology and Venereology, Mafraq Hospital, Abu Dhabi, United Arab Emirates, ${ }^{2}$ Medical Student, Faculty of Medicine and Health Science, UAE University, Al Ain, United Arab Emirates, ${ }^{3}$ Medical Student, Faculty of Medicine and Health Science, Sharjah University, Sharjah, United Arab Emirates
\end{abstract}

Corresponding author: Dr. Mariam Al Zaabi, E-mail: dr_mariam01@hotmail.com

\begin{abstract}
Prurigo nodularis (PN) is chronic unbearable inflammatory skin disease. Although it was described before a century, not many studies have been conducted regarding the systemic treatment of prurigo nodularis. A 64-year-old male patient has moderate to severe atopic dermatitis superimposed by disseminated pruritic nodules over the trunk and extremities. In spite of topical treatment and Phototherapy, patient condition was deteriorating. Therefore, the patient was treated with multimodalities including high potency topical steroid, intravenous antihistamine, cyclosporine and omalizumab without improvement. Thus the patient has been treated with methotrexate which led to remarkable improvement. Management of prurigo nodularis is often challenging as the etiology of PN in the majority of the cases is unknown. Conservative treatments are often inefficient. This case proves the efficacy of methotrexate in the management of prurigo nodularis.
\end{abstract}

Key words: Refractory prurigo; Methotrexatep; Pruritic nodules; Systemic therapy

\section{INTRODUCTION}

Prurigo nodularis PN is chronic intolerable inflammatory skin disease. It results from chronic pruritus. The term prurigo is Latin and means itching. PN was initially described by J.N. Hyde on 1909. Although it was described before 105 years, still the pathogenesis unclear and not many studies have been conducted regarding the systemic treatment of prurigo nodularis. The diagnosis and therapy of chronic pruritus and prurigo nodularis require multidiscipline [1].

\section{CASE REPORT}

A 64-year-old male patient has moderate to severe atopic dermatitis since 2007, and had been treated for a long time with topical and systemic steroids as well as topical calcineurin inhibitor such as pimecrolimus and tacrolimus, which lead to transit improvement in patient's condition. However, 2 years later patient developed disseminated pruritic nodules over the trunk and extremities. Clinically and histologically the diagnosis of prurigo nodularis has been established.
He was treated with high potency topical steroid and oral antihistamines medication without improvement. Intralesional injection of triamcinolone did not show any benefit. Thus patient was intensively investigated, his complete blood count test (CBC) was not significant, his urea and electrolytes as well as creatinine levels were within normal limits. His liver enzymes were normal. Thyroid function test (TFT) was not significant. Total $\mathrm{IgE}$ was 769 which is high whereas specific IgE were irrelevant. Prick test for pollen and food allergen and standard patch test were insignificant. Autoimmune antibodies including ANA, Pemphigus and Pemphigoid and Duhring antibodies have been investigated and were negative. CD4/CD8 ratio was normal.

In spite of topical treatment as well as Phototherapy (including UVB and PUVA), patient's condition was deteriorating. Therefore, the patient was treated with multimodalities including high potency topical steroid, intravenous antihistamine and cyclosporine. However, cyclosporin has been ceased due to uncontrolled hypertension and unbearable gastrointestinal symptoms. Moreover, his symptoms were reluctant to the systemic

\footnotetext{
How to cite this article: Al Zaabi M, Al Suwaiji M, Nasir M. Methotrexate for refractory prurigo nodularis. Our Dermatol Online. $2017 ; 8(1): 40-42$.

Submission: 30.03.2016; Acceptance: 31.05.2016

DOI:10.7241/ourd.20171.10
}

(c) Our Dermatol Online 1.2017 
therapy with omluzimumab. Thus the patient has been treated with methotrexate $15 \mathrm{mg}$ subcutaneous weekly, remarkable improvement has been noticed 3 months following the onset of treatment. Consequently the dose of Methotrexate was gradually reduced, and then discontinued following complete resolution of the pruritus and prurigo nodules (Figs. 1 and 2).

\section{DISCUSSION}

\section{Definition}

Prurigo nodularis is chronic enervating inflammatory skin disorder that may affect the entire body. This disease can occur in all age groups but it primarily affects adults. The exact etiology of prurigo nodularis is still unknown. It results from chronic scratching due to many etiologies, such as dermatological disorders as xerosis or atopic dermatitis and systemic diseases for instance hyperthyroidism, hepatic or renal dysfunction,

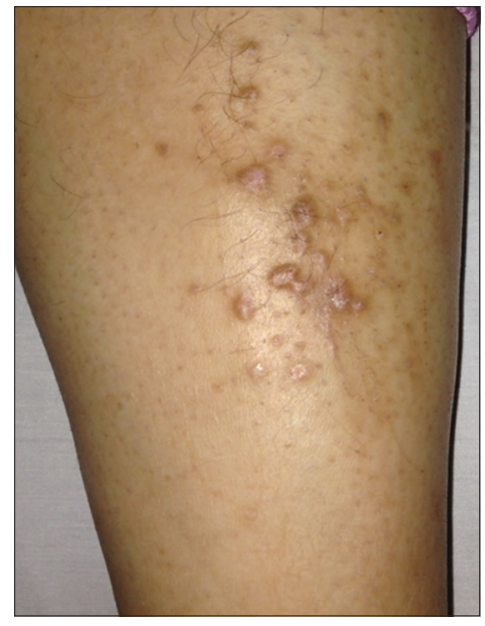

Figure 1: Before treatment with methotrexate.

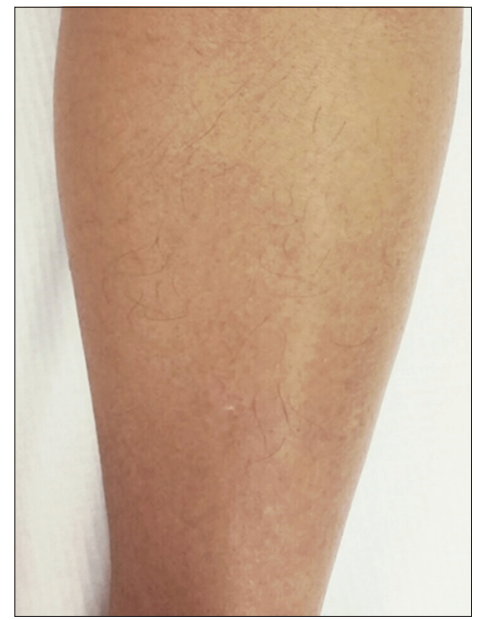

Figure 2: 8 months following discontinuation of methotrexate.
Lymphoma and iron deficiency. Emotional distress and psychological illnesses are also common contributing factors [2-4].

The lesions of prurigo nodularis vary in quantity and morphology. The eruption can be erythematous, brown or skin color. The lesions present usually in hard dome shaped, papular or nodular appearance that are excoriated and have central scale or crust. Those nodules present in a symmetric distribution with predominance on the extensor surfaces of the upper and lower limbs. Prurigo nodularis can be diagnosed clinically as it has characteristic morphology. Beside that histological investigation is a useful confirmatory diagnostic tool. Further investigations to rule out any underlying systemic causes of pruritus are crucial such as $\mathrm{CBC}$, liver function test, and creatinine and TFT [4-7].

\section{Pathogenesis}

The exact pathophysiology of PN is not totally clear. Recent studies proposed neurogenic mechanisms as dermal hyperplasia and epidermal hypoplasia of sensory nerve fibers have been documented. This supported by effectiveness of thalidomide or even gabapentin in the management of PN. In addition, higher levels of the novel pruritic cytokine IL 31 were recently found in the skin of patients with prurigo nodularis than other pruritic skin disease. Moreover, latest studies have revealed that mast cells play a crucial role in the genesis of pruritus in $\mathrm{PN}$. It has been observed that mast cells in PN lesions present in an abundant quantity adjacent to peripheral nerves in patients with $\mathrm{PN}$ and have distinctive morphology such as an enlarged cell body and a dendritic shape compared with the round or elongated figure observed in the normal skin. The PN mast cells also have an abundant cytoplasm with a reduced amount of granules, proposing that many of the granules have been released into the surrounding tissue. Mast cells in PN have been observed to produce more nerve growth factor (NGF) in the lesional skins leading to neural hyperplasia. Subsequently, this neural hyperplasia leads to intense pruritus. Apart from neural hyperplasia, there are other mast cell products that may contribute to pruritus in $\mathrm{PN}$; these include histamine, tryptase, prostaglandins, and interleukins. Thus the pathogenesis of PN seems to be regulated by immunological neuronal plasticity [7-9].

\section{Treatment}

Prurigo nodularis is often refractory to various therapeutic regimens. Optimal skin hydration through 
regular use of emollients is the mainstay of treatment in pruritus, as emollients enhance the skin barrier function and prevent entry of irritants. The topical treatments of PN include antipruritic agent such as menthol and anesthetic agent such as pramoxine. Topical capsaicin and calcipotriol have been reported as effective therapies. Potent and super- potent corticosteroid can also be effective due to their anti-inflammatory properties. Intralesional corticosteroids such as dexamethasone or triamcinolone suspensions may be effective but they are unpractical if there are numerous lesions. Cryotherapy has been used but depigmentation and scarring can occur. For disseminated lesions, phototherapy with UVB or PUVA can be administered. Systemic treatments used for PN includes antihistamine medication, anti-depressants such as amitriptyline or doxepin, oral steroids and naltrexone. In sever refractory cases cyclosporine, azathioprine, methotrexate, thalidomide and Immunoglobulin has been reported as efficient $[10,11]$.

Methotrexate is a folic acid antagonist commonly used in the management of inflammatory, autoimmune and malignant disorders. The anticancer property of MTX is well described; it suppresses the key enzymes in the biosynthesis of purines and pyrimidines, thereby reducing malignant cell proliferation and turnover. Besides that, it has anti-inflammatory effect but it is poorly understood. The most probable anti-inflammatory effect of MTX is enhanced extracellular concentrations of adenosine which has potent anti-inflammatory activity. Adenosine interferes with pro-inflammatory consequences of classical macrophage activation, leading to suppression of cytokine/chemokine production such as IL6, IL12, tumor necrosis factor TNF and interferon $\mathrm{y}$. In addition, recruitment and activation of Neutrophils is impaired by Adenosine. This elucidates the efficiency of MTX in the management of atopic dermatitis and its associated PN $[8,9,12]$.

\section{CONCLUSION}

Management of prurigo nodularis is often challenging as the etiology of PN in the majority of the cases is unknown. Conservative treatments such as topical corticosteroids antipruritic agents and phototherapy are often inefficient. This case proves the efficacy of methotrexate in the management of prurigo nodularis, however further studies should be conducted to assess the long term effectiveness of MTX in different age groups.

\section{Consent}

The examination of the patient was conducted according to the Declaration of Helsinki principles.

\section{REFERENCES}

1. Ständer S, Mettang T. Prurigo nodularis: A puzzle for more than 100 years. Hautarzt. 2014;65:672-3.

2. Schedel F, Schürmann C, Metz D, Ständer S. Prurigo. Clinical definition and classification. Hautarzt. 2014;65:684-90.

3. Fostini A, Girolomoni G, Tessari G. Prurigo nodularis: an update on etiopathogenesis and therapy. J Dermatology Treat. 2013;24:458-62.

4. Mettang T, Vonend A, Raap U. Prurigo nodularis: its association with dermatoses and systemic disorders. Hautarzt. 2014;65:697-703.

5. Böhme T, Heitkemper T, Mettang T, Phan NQ, Ständer S. Clinical features and prurigo nodularis in nephrogenic pruritus. Hautarzt. 2014;65:714-20.

6. Tan WS, Tey HL. Extensive prurigo nodularis: characterization and etiology. Dermatology. 2014;228:276-80.

7. Raap U, Günther C. Pathogenesis of prurigo nodularis. Hautarzt. 2014;65:691-6.

8. György H, Bruce C. Regulation of Inflammation by Adenosine. Front Immunol. 2013;4:85.

9. Spring P, Gschwind I, Gilliet M. Prurigo nodularis: Retrospective study of 13 cases managed with methotrexate. Clin Exp Dermatol. 2014;39:468-73.

10. Zeidler C, Ständer S. Therapy of prurigo nodularis. Hautarzt. 2014;65:709-13. 11- Feldmeyer L, Werner S, Kamarashev J, French LE, Hofbauer GF.Atopic prurigo nodularis responds to intravenous immunoglobulins. Br J Dermatol. 2012;166:461-2.

11. Ashimav D. Oral Ketotifen and Topical Antibiotic Therapy in the Management of Pruritus in Prurigo Nodularis: A Randomized, Controlled, Single-Blind, Parallel Study. Ind J Dermatol. 2013;58:355-9.

12. Chan ES, Cronstein BN. Mechanisms of action of methotrexate. Bull Hosp Jt Dis. 2013;71:5-8.

Copyright by Mariam Al Zaabi, et al. This is an open access article distributed under the terms of the Creative Commons Attribution License, which permits unrestricted use, distribution, and reproduction in any medium, provided the original author and source are credited.

Source of Support: Nil, Conflict of Interest: None declared. 